\title{
Self-Dual Yang-Mills Fields and Deformations of Algebraic Curves
}

\author{
D. A. Korotkin \\ Department of Mathematics, Leningrad Institute of Aircraft Instrumentation, \\ Gertzena, 67, SU-190000 Leningrad, USSR
}

Received March 19, 1990

\begin{abstract}
Recently it has been shown that the methods of algebraic geometry first used for finding periodic and almost periodic solutions of $\mathrm{KdV}, \mathrm{HSh}, \mathrm{SG}$ and other equations [11-13] may be successfully applied to study the solutions of nonlinear equations with a variable spectral parameter in associated zero-curvature representation. In this work following [20] this treatment is extended to the case of the self-duality equation. It seems to be the first example of a four-dimensional non-linear equation solvable by the method of finite-gap integration. Two broad classes of finite-gap solutions for each $-S U(2)$ and $S U(1,1)$ gauge groups are constructed in terms of multidimensional theta-functions. The dynamics of the solutions is given by the movement of the hyperelliptic curve with moving branch points and a divisor of the poles in the moduli space of algebraic curves. In the general case our solutions have no periodicity property. We show how oneinstanton solution and $5 \mathrm{~N}$-parametric t'Hooft family of instantons may be obtained by the degeneration of general formulae.
\end{abstract}

\section{Introduction}

The problem of obtaining and investigating particle-like (soliton) solutions of the field equations attracted in the 70's a great interest of many mathematicians and physicists. It is impossible to give here even a brief review of the main results in the field (for history and references see [1-3]); we will mention only results closely related to the subject of this paper.

Belavin and Zakharov [4] obtained a U-V pair for the self-duality equation and, therefore, it appeared possible to apply the inverse scattering technique to this equation. However, one of the most important problems in the field - the description of all multi-instanton configurations was solved in a remarkable paper [5] by a different algebraic-geometric AHDM method. Nahm's modification of this method allowed to solve another problem - the classification of all multimonopole configurations. Despite very good results of this method some important problems remain unsolved - for example, effective description of the 
moduli spaces of multi-instanton solutions with topological charge more than three.

In comparison with the algebraic-geometric approach of AHDM (or AHDMN) the results of applying the traditional inverse scattering method (ISM) based on the Riemann-Hilbert problem [6-10] to this subject appear not so advanced. For four-dimensional fields this method allowed to obtain only a $5 \mathrm{~N}$ parametric 'tHooft instanton family $[7,8]$ instead of a general $8 \mathrm{~N}-3$-parametric family; for three-dimensional fields the description of a $4 N-1$-parametric general multimonopole configuration by the standard "dressing" technique [6] appears not very effective too.

Simultaneously since 1974 the theory of finite-gap periodic and almost periodic solutions for some non-linear differential equations integrable by IST (Korteweg de Vries (KdV), non-linear Schrödinger (NSh), Sine-Gordon (SG) and others) has been developed. This theory began with the works of Novikov, Dubrovin, Matveev, and Its in application to KdV. Further in extending to other equations this method has been greatly improved. The theory of finite-gap integration where the main role is played by the matrix function with the special analytical properties on the algebraic curve known as the Baker-Akhiezer function has been developed by Krichever (see Reviews [11-13]). Reformulation of this method in terms of the generalized Riemann-Hilbert problem was proposed in $[14,15]$. The dynamics of corresponding solutions is defined by the linear trajectory on the Jacobi manifold of the fixed algebraic curve. If the curve is degenerated into a curve of zero genus the periodic and almost periodic solutions are transformed into localized - multisoliton solutions. This method for obtaining multisoliton solutions seems more effective in comparison with the "dressing" technique or Backlund transformation.

Further development of the method of finite-gap integration [16-18] allowed to construct a new broad class of algebraic-geometric solutions of axisymmetric stationary Einstein and Einstein-Maxwell systems and some other equations with "variable spectral parameter" [19]. The main features of these solutions are the following: in contrast to ordinary finite-gap solutions of KdV, NSh, SG and others they are not periodic or almost periodic but localized as the degenerated - soliton solutions; the dynamics is set by the trajectory in the moduli space of algebraic curves.

This technique was generalized in [20] to construct the solutions in terms of Riemann theta-functions for $S U(2)$ and $S U(1,1)$ self-duality equations. The reduction to the stationary axisymmetric vacuum Einstein equation is also discussed therein.

This work is devoted to obtaining one more class of finite-gap solutions for each $-S U(2)$ and $S U(1,1)$ case. To illustrate possible applications of our approach we derive a one-instanton solution and a $5 \mathrm{~N}$-parametric 'tHooft family of instantons by degeneration of general formulae. It is quite natural to suppose that some alternative (which may be more effective in comparison with AHDM construction) description of general $8 N-3$-parametric instanton family may be obtained in this way. Another possible application of our approach may be to obtain a more convenient formulae for multimonopole solutions. Finally, a very interesting question is a mathematical and physical interpretation of nondegenerated solutions.

It should be noted also that some aspects of the application of the finite-gap technique to the self-duality equation were considered in $[21,22]$, but the explicit 
formulae for solutions were not obtained therein. Application of the finite-gap method to Nahm's equations is considered in detail in the recent work [23].

The paper is organized as follows: Sect. 2 includes some general facts and the formulation of the Riemann-Hilbert problem for the matrix Baker-Ahieser-type function. Section 3 includes the explicit construction in terms of Riemann thetafunctions of two broad classes of solutions for $S U(2)$ case and two classes for the $S U(1,1)$ case. The corresponding classes of $S U(2)$ and $S U(1,1)$ solutions seem to be connected by Backlund transformation by Corrigan et al. [24]. In Sect. 4 we show how to derive from general formulae one-instanton solution and 'tHooft family of instantons by the degeneration of the associated algebraic curve.

\section{Generalized Riemann-Hilbert Problem for the Matrix Baker-Akhiezer-Type Function}

The self-duality equations for Yang-Mills field may be written in the form

$$
\boldsymbol{F}_{\mu \nu}(x)=* \boldsymbol{F}_{\mu v}(x),
$$

where $* \boldsymbol{F}_{\mu \nu} \equiv 1 / 2 \varepsilon_{\mu \nu \alpha \beta} \boldsymbol{F}^{\alpha \beta}, \varepsilon_{\mu \nu \alpha \beta}-$ completely antisymmetric tensor and $\varepsilon_{1234}=1, \alpha$, $\beta, \mu, v=1, \ldots, 4, x=\left(x_{1}, \ldots, x_{4}\right) \in \mathbb{R}^{4}$,

$$
\boldsymbol{F}_{\mu \nu}(x)=\partial_{\mu} \boldsymbol{A}_{v}(x)-\partial_{v} \boldsymbol{A}_{\mu}(x)+\left[\boldsymbol{A}_{\mu}(x), \boldsymbol{A}_{v}(x)\right],
$$

$A_{\mu} \in S U(2)$ or $S U(1,1)$.

Let us introduce complex coordinates $y=x_{1}+i x_{2}, z=x_{3}+i x_{4}, \bar{y}, \bar{z}$. Then (2.1) may be written in the form

$$
\begin{aligned}
& F_{y z}=F_{\bar{y} \bar{z}}=0, \\
& F_{y \bar{y}}=F_{z \bar{z}}=0 .
\end{aligned}
$$

To satisfy (2.2a) it is necessary to suppose that $A_{y}=-\sigma D_{y} D^{-1} \sigma, A_{\bar{y}}=D^{-1+} D_{\bar{y}}^{+}$, $A_{z}=-\sigma D_{z} D^{-1} \sigma, A_{\bar{z}}=D^{-1+} D_{\bar{z}}$, where in $S U(2)$ case $\sigma=I$ (unit matrix), in $S U(1,1)$ case $\sigma=\sigma_{3}\left(\sigma_{i}, i=1,2,3\right.$ - Pauli matrices); $\operatorname{det} D=1$. Then (2.2b) takes the form:

$$
\left(J_{y} J^{-1}\right)_{\bar{y}}+\left(J_{z} J^{-1}\right)_{\bar{z}}=0 \text {, }
$$

where $J=D \sigma D^{+}$-hermitian matrix with determinant 1 in the $S U(2)$ case and -1 in the $S U(1,1)$ case.

Equation (2.3) is an integrability condition of the following linear system:

$$
\left\{\begin{array}{l}
\left(\lambda \partial_{\bar{z}}+\partial_{y}\right) \Psi=B_{y} \Psi \\
\left(-\lambda \partial_{\bar{y}}+\partial_{z}\right) \Psi=B_{z} \Psi,
\end{array}\right.
$$

where $B_{y}=J_{y} J^{-1}, B_{z}=J_{z} J^{-1} ; \lambda \in \mathbb{C}$-spectral parameter; $\Psi(\lambda, y, \bar{y}, z, \bar{z})$-matrix $2 \times 2$ function. System (2.4) may be obtained by a simple gauge transformation [8] from $\mathrm{U}-\mathrm{V}$ pair of Belavin and Zakharov [4].

We shall define the Baker-Akhiezer-type $2 \times 2$ matrix function corresponding to the linear system (2.4) first axiomatically, by imposing on $\Psi$ the following conditions A-F:

A. $\Psi(\lambda, y, \bar{y}, z, \bar{z}): \mathbb{C} \times \mathbb{R}^{4} \rightarrow \operatorname{Mat}(2 \times 2)$ is holomorphic and invertible on $\mathbb{C}$ (for the fixed values of $(y, z)$ except the points described in the conditions $\mathbf{B}-\mathbf{E}$ given below. 
B. $\Psi(\lambda)$ has regular singularities at the points $a_{1}(y, \bar{y}, z, \bar{z}), \ldots, a_{n}(y, \bar{y}, z, \bar{z}), a_{i} \in \mathbb{C}$ i.e. $\Psi(\lambda)$ has the following behaviour at the points $a_{i}$ :

$$
\Psi(\lambda)_{\lambda \approx a_{i}} \Psi(\lambda)\left(\lambda-a_{i}\right)^{T_{i}} C_{i}, \quad i=1, \ldots, n,
$$

where $C_{i}$ - constant, $T_{i}$ - diagonal constant matrices; $\Psi(\lambda)$ is holomorphic and invertible in the neigbourhood of $a_{i}$;

$$
\left(\tau^{T_{i}}\right)_{k l} \equiv \tau^{\left(T_{i}\right)_{k l}}
$$

$\left(\tau=\lambda-a_{i}\right.$-local parameter at $\left.a_{i}\right)$. All functions $a_{i}(y, \bar{y}, z, \bar{z})$ have to solve the following "pole system":

$$
\left.\begin{array}{r}
a a_{\bar{z}}+a_{y}=0 \\
-a a_{\bar{y}}+a_{z}=0
\end{array}\right\}
$$

$\left(a=a_{1}, \ldots, a_{n}\right)$. If the elements of matrix $T_{i}$ are non-integer then $a_{i}$ is the end of the cut on $\mathbb{C}$ (see condition $\mathbf{C}$ ).

C. Let $\left\{K_{j}\right\}, K_{j} \subset \mathbb{C}, j=1, \ldots, \imath$ be some system of oriented paths with the following property: boundary values of the function $\Psi(\lambda)$ are related along the paths $K_{j}$ by the equations:

$$
\left.\Psi_{-}(\lambda)\right|_{K_{j}^{-}}=\left.\Psi_{+}(\lambda)\right|_{K_{j}^{+}} G_{j}(\lambda), \quad j=1, \ldots, \imath,
$$

where the conjugation matrices $G_{j}$ are independent upon $y, \bar{y}, z, \bar{z}$; the ends of the paths $K_{j}$ solve pole system (2.5).

D. The behaviour of $\Psi(\lambda)$ at $\lambda=\infty$ is following:

$$
\Psi(\lambda, y, \bar{y}, z, \bar{z})_{\lambda \sim \infty} f_{1}(y, z) f_{2}(\lambda, \lambda y-\bar{z}, \lambda z+y)\left(1+O\left(\frac{1}{\lambda}\right)\right),
$$

where $f_{1,2}$ - sufficiently smooth matrix functions (Normalization condition).

E. The behaviour of $\Psi(\lambda)$ at $\lambda=0$ is following:

$$
\Psi(\lambda, y, \bar{y}, z, \bar{z})_{\lambda} \approx 00(y, \bar{y}, z, \bar{z}) h(\lambda, \lambda y-\bar{z}, \lambda z+y)(1+O(\lambda)),
$$

where $J$-invertible matrix; $h$ plays the same role as function $f_{2}$ in the previous point.

Conditions B-E may be summarized as follows: $\Psi(\lambda)$ has only such particular points (poles, branch points, branch cuts and so on) that the logarithmic derivatives $\left(\lambda \Psi_{\bar{z}}+\Psi_{y}\right) \Psi^{-1}$ and $\left(-\lambda \Psi_{\bar{y}}+\Psi_{z}\right) \Psi^{-1}$ are holomorphic at these points.

F. Matrix $J(y, \bar{y}, z, \bar{z})$ from $\mathbf{E}$ is hermitian and $\operatorname{det} J=1$ (in $S U(2)$ case) or $\operatorname{det} J=-1$ (in $S U(1,1)$ case).

It is easy to prove the following important statement:

Theorem. Let $\Psi(\lambda, y, \bar{y}, z, \bar{z})$ be some $2 \times 2$ matrix function satisfying the conditions A-F. Then the matrix function $J(y, \bar{y}, z, \bar{z})$ solves Eq. (2.4).

The proof may be obtained by applying the Liouville theorem to logarithmic derivatrives of $\Psi$ which are holomorthic on $\mathbb{C}$, in full analogy with the proof of the similar statement in the case of a stationary axisymmetric Einstein equation [16]. 
Now to obtain the solution of (2.3) it is sufficient to construct effectively some function $\Psi$ corresponding to generalized Riemann data $\Lambda$ :

$$
\Lambda=\left\{a_{i}, T_{i}, C_{i}, i=1, \ldots, n, G_{k}(\lambda), \lambda \in K_{k}, k=1, \ldots, l ; f_{1,2}, h\right\} .
$$

We shall obtain finite-gap or algebraic-geometric solutions of self-duality equations, expressed in terms of multidimensional Riemann theta-functions corresponding to some special Riemann data.

\section{Explicit Construction and multidimensional Theta-Functions}

Let us consider a hyperelliptic algebraic curve $\mathfrak{L}$ of genus $2 \mathfrak{g}-1$ defined by the equation

$$
\omega^{2}=\prod_{i=0}^{2 \mathfrak{g}-1}\left(\lambda-E_{i}\right)\left(\lambda-F_{i}\right),
$$

where all branch points $E_{i}(y, \bar{y}, z, \bar{z}), F_{i}(y, \bar{y}, z, \bar{z}), i=0, \ldots, 2 \mathfrak{g}-1$ are solutions of (2.5). To satisfy condition $\mathbf{D}$ it is necessary to assume that $\mathbb{R}$ is invariant under the anti-involution $\tau: \lambda \rightarrow-(\bar{\lambda})^{-1}$ on every sheet of $\mathfrak{L}$ (system (2.5) is invariant under the substitution $\left.a \rightarrow-(\bar{a})^{-1}\right)$. Therefore let us suppose that $\tau E_{i}=E_{i+g}, \tau F_{i}=F_{i+g}$; branch cuts $\left[E_{i}, F_{i}\right]$ lie inside and branch cuts $\left[E_{i+g}, F_{i+g}\right]$ - outside of the circle $|\lambda|=1, i=0, \ldots, g-1$ (see Fig. 1).

Denote by $*$ the involution on $\mathfrak{L}$ interchanging the sheets: $*:(\omega, \lambda) \rightarrow(-\omega, \lambda)$. To define the matrix function $\Psi(P)(P=(\omega, \lambda))$ on the first sheet of $\mathfrak{Q}$ we shall use the ansatz

$$
\Psi(P)=T\left(\begin{array}{ll}
\varphi(P) & \varphi^{*}(P) \\
\psi(P) & \psi^{*}(P)
\end{array}\right) \equiv T \Phi(P),
$$

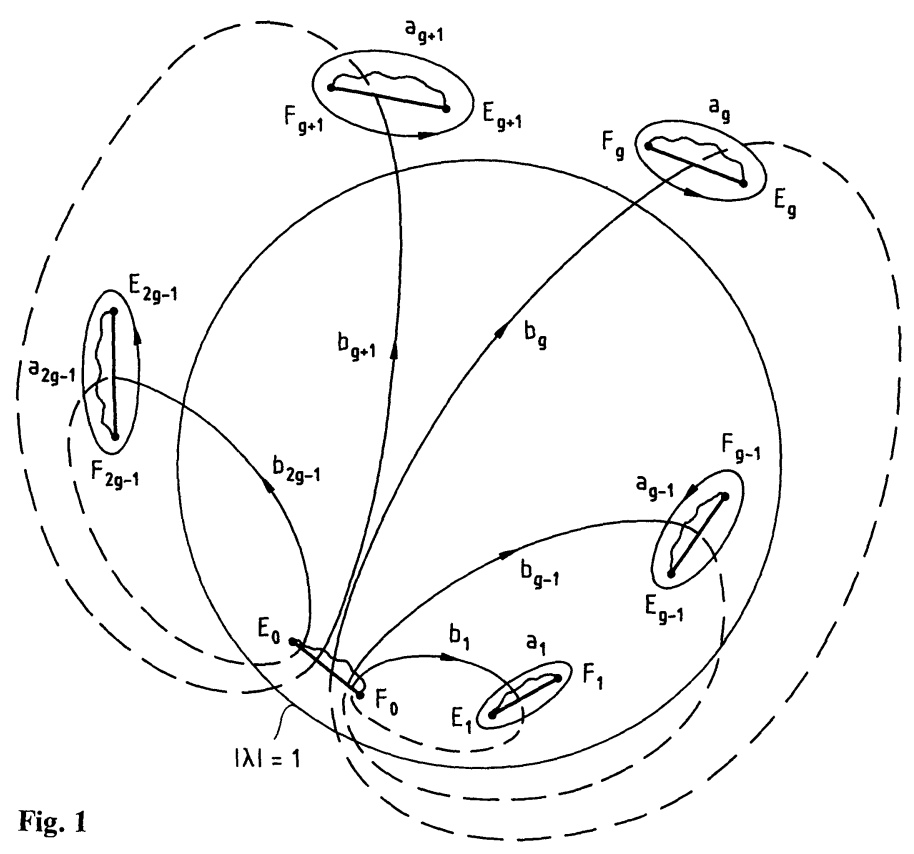


widely used in the theory of finite-gap integration, where $P$ lies on the first sheet of $\mathfrak{L}, \varphi$ and $\psi$ - two scalar functions on $\mathfrak{L}, \varphi^{*}, \psi^{*}(P) \equiv \varphi, \psi\left(P^{*}\right)$; multiplication on $\lambda$-independent matrix function $T$ is necessary to satisfy condition $\mathbf{D}$; the notation $\Phi(P)$ is introduced for convenience.

Now let's introduce the standard objects of finite-gap construction. Canonical basis of cycles $a_{i}, b_{i}, i=1, \ldots, 2 \mathfrak{g}-1$ is chosen as shown in Fig. 1 . Let $d U_{i}(P)$, $i=1, \ldots, 2 \mathfrak{g}-1$ be the dual basis of holomorthic abelian differentials on $\mathfrak{Q}$ with the normalization $\oint_{a_{i}} d U_{k}=\delta_{i k}$. The matrix of $\delta$-periods $\mathbb{B}_{i k}$ and Abel mapping $U(P)$ are defined by the formulae:

$$
\mathbb{B}_{i k}=\oint_{b_{i}} d U_{k}(P) ; \quad U_{i}(P)=\int_{P_{0}}^{P} d U_{i}, \quad i, k=1, \ldots, 2 \mathfrak{g}-1 .
$$

The Riemann theta-function is defined as follows:

$$
\begin{gathered}
\vartheta(\boldsymbol{x} \mid \mathbb{B})=\sum_{\boldsymbol{m} \in \mathbb{Z}^{\mathfrak{2}-1}} \exp \{\pi i\langle\mathbb{B} \boldsymbol{m}, \boldsymbol{m}\rangle+2 \pi i\langle\boldsymbol{x}, \boldsymbol{m}\rangle\}, \\
\boldsymbol{x} \in \mathbb{C}^{2 \mathfrak{g}-\mathbf{1}},\langle\boldsymbol{x}, \boldsymbol{m}\rangle \equiv \boldsymbol{x}_{\mathbf{1}} \boldsymbol{m}_{\mathbf{1}}+\ldots+\boldsymbol{x}_{\mathbf{2 g}-\mathbf{1}} \boldsymbol{m}_{\mathbf{2 g}-\mathbf{1}} .
\end{gathered}
$$

Since in this paper we will consider only possible applications to instantontype (after degeneration) solutions, it is sufficient here to choose functions $f_{1,2}$ and $h$ in the following form:

$$
\begin{gathered}
f_{1}(y, z)=C ; \quad f_{2}(\lambda, \lambda y-\bar{z}, \lambda z+\bar{y})=\operatorname{diag}\left(\lambda^{\alpha}, \lambda^{-\alpha}\right), \\
h(\lambda, \lambda y-\bar{z}, \lambda z+\bar{y})=\operatorname{diag}\left(\lambda^{ \pm \alpha}, \lambda^{\mp \alpha}\right),
\end{gathered}
$$

where $\alpha \in \mathbb{R}$-arbitrary constant, $C$-constant matrix.

If we would like to consider in our approach (after degeneration) the monopole-type solutions; then it would be necessary to take $f_{1,2}$ and $h$ in the exponential form; however, for the explicit construction to be more transparent, we do not consider here this generalization of the present method because it doesn't include any new ideas.

Now we shall consider explicit constructions of different classes of our solutions:

a. $S U(2) \tau$-Solutions. We can directly construct two classes of $S U(2)$ solutions. The first class corresponds to the case when the analytical properties of functions $\varphi$ and $\psi$ from (3.1) on $\mathfrak{L}$ are invariant under anti-involution $\tau$, and the second class under anti-involution $\tau *$. Therefore we will call the solutions of the first class " $\tau$-solutions" and the solutions of the second class " $\tau *$-solutions."

In the present case let us define functions $\varphi(P)$ and $\psi(P)$ by the formulae:

$\varphi(P)=\frac{\vartheta\left(\boldsymbol{U}(P)-\boldsymbol{U}(\boldsymbol{D})-\boldsymbol{U}\left(D_{0}\right)+\boldsymbol{U}\left(Q_{\varphi}\right)+\boldsymbol{B}+\boldsymbol{b}-\boldsymbol{K}\right)}{\vartheta(\boldsymbol{U}(P)-\boldsymbol{U}(\boldsymbol{D})-\boldsymbol{K})} \exp \left\{W_{Q_{\varphi} D_{0}}(P)+W(P)+w(P)\right\}$

$\psi(P)=\frac{\vartheta\left(\boldsymbol{U}(P)-\boldsymbol{U}(\boldsymbol{D})-\boldsymbol{U}\left(D_{0}\right)+\boldsymbol{U}\left(Q_{\psi}\right)+\boldsymbol{B}+\boldsymbol{b}-\boldsymbol{K}\right)}{\vartheta(\boldsymbol{U}(P)-\boldsymbol{U}(\boldsymbol{D})-\boldsymbol{K})} \exp \left\{W_{Q_{\psi} D_{0}}(P)+W(P)+w(P)\right\}$,

where $D_{0}+\boldsymbol{D}=D_{0}+D_{1}+\ldots+D_{2 \mathfrak{g}-1}$ is a non-special divisor invariant under antiinvolution $\tau:\left(D_{0}+\boldsymbol{D}\right)^{\mathfrak{\tau}}=D_{0}+\boldsymbol{D}$; all functions $D_{i}(y, \bar{y}, z, \bar{z}), i=0, \ldots, 2 \mathfrak{g}-1$ are solutions of (2.5); $\boldsymbol{K}$ is a vector of Riemann constants; $Q_{\varphi}$ and $Q_{\psi}$ are arbitrary 
points on $\mathfrak{E} ; W_{Q_{\varphi} D_{0}}(P)$ and $W_{Q_{\varphi} D_{0}}(P)$ are normalized (all $a$-periods are zero) abelian integrals of the third kind with the residue +1 at $Q_{\varphi}$ (respectively $Q_{\psi}$ ) and -1 at $D_{0} ; 2 \pi i\left(U\left(Q_{\varphi}\right)-U\left(D_{0}\right)\right)$ and $2 \pi i\left(U\left(Q_{\psi}\right)-U\left(D_{0}\right)\right)$ are their $b$-period vectors. A normalized abelian integral $W(P)$ is a third kind integral with poles at $\infty^{1}, \infty^{2}, O^{1}$, $O^{2}$ and residue $\alpha,-\alpha, \alpha,-\alpha$ respectively; $2 \pi i \boldsymbol{B}=\alpha\left(\boldsymbol{U}\left(\infty^{1}\right)-\boldsymbol{U}\left(\infty^{2}\right)+\boldsymbol{U}\left(O^{1}\right)\right.$ $\left.-U\left(O^{2}\right)\right)$ - its vector of $b$-periods. The integral $w(P)$ is an arbitrary linear combination with the constant coefficients of normalized third kind abelian integrals whose poles all satisfy $(2.5) ; w(P)$ must be real, i.e. $\bar{w}\left(P^{\tau}\right)=w(P) ; 2 \pi i b$ is a vector of $b$-periods of $w(P)$.

The function $\Psi(\lambda)$ defined by (3.1) on the first sheet of $\mathfrak{Q}$ satisfies conditions A-E with some Riemann-Hilbert problem data which may be easily written in full analogy with [16-18].

Consider condition $\mathbf{F}$. Due to the invariance of all poles of $\varphi$ and $\psi$ under antiinvolution $\tau$ if we assume $Q_{\varphi}=Q_{\psi}^{\tau}$, then

$$
\bar{\varphi}\left(P^{\tau}\right) / \psi(P)=\text { const } \equiv c(y, \bar{y}, z, \bar{z}) .
$$

Note that the variation of the poles $Q_{\varphi}$ and $Q_{\psi}$ is equivalent to the variation of the basis in two-dimensional linear space $S(\varphi, \psi)$ of linear combinations $a \varphi+b \psi$ $(a, b \in \mathbb{C})$.

The matrix function $\Phi(P)$ defined by (3.1) and (3.4) has the following local behaviour near points $O^{1}$ and $\infty^{1}$ :

where

$$
\begin{gathered}
\Phi(\lambda)_{\lambda \sim 0^{1}} \Phi_{0}(y, \bar{y}, z, \bar{z})\left(\begin{array}{cc}
\lambda^{\alpha} & 0 \\
0 & \lambda^{-\alpha}
\end{array}\right)(1+O(\lambda)), \\
\Phi(\lambda)_{\lambda \sim \infty^{1}} \Phi_{\infty}(y, \bar{y}, z, \bar{z})\left(\begin{array}{cc}
\lambda^{-\alpha} & 0 \\
0 & \lambda^{\alpha}
\end{array}\right)\left(1+O\left(\lambda^{-1}\right)\right),
\end{gathered}
$$

$$
\Phi_{0}=\left(\begin{array}{cc}
\varphi_{1}^{0} & \varphi_{2}^{0} \\
\psi_{1}^{0} & \psi_{2}^{0}
\end{array}\right), \quad \Phi_{\infty}=\left(\begin{array}{cc}
\varphi_{1}^{\infty} & \varphi_{2}^{\infty} \\
\psi_{1}^{\infty} & \psi_{2}^{\infty}
\end{array}\right) .
$$

Now we choose matrix $C$ from (3.3) equal to $\sigma_{2}$; then from (3.1), (3.3), (3.4) one obtains:

$$
\Psi(\lambda)=\sigma_{2} \Phi_{\infty}^{-1} \Phi(\lambda) .
$$

The corresponding function $J$ takes the form

$$
J(y, \bar{y}, z, \bar{z})=\sigma_{2} \Phi_{\infty}^{-1} \Phi_{0} .
$$

Note the following simple fact: if $J$ is a solution of (2.3) but $\operatorname{det} J \neq \pm 1$ then the "physical" solution $J_{\mathrm{ph}}=( \pm \operatorname{det} J)^{1 / 2} J$ satisfies $(2.3)$ too and $\operatorname{det} J_{\mathrm{ph}}=1$. In our case

$$
J_{\mathrm{ph}}=\left(\operatorname{det} X_{\infty} / \operatorname{det} X_{0}\right)^{1 / 2} \sigma_{2} X_{\infty}^{-1} X_{0} .
$$

From (3.5) we see that

$$
\bar{\varphi}_{1,2}^{\infty}=c \psi_{1,2}^{0},
$$

and $\operatorname{det} \Phi_{\infty}=-(c / \bar{c}) \overline{\operatorname{det}}_{0}$. After simple calculations one can verify that $J_{\mathrm{ph}}$ is hermitian and, therefore, gives a solution of the $S U(2)$ self-duality equation.

To obtain from (3.8) explicit formulae for gauge potentials $A_{\mu}$ one needs to represent $J_{\mathrm{ph}}$ as $D D^{+}$where $D \in S L(2, \mathbb{C})$. From (3.8) and (3.9) we find

$$
D=\left(\varphi_{1}^{0} \psi_{2}^{0}-\varphi_{2}^{0} \psi_{1}^{0}\right)^{-1 / 2}\left(\begin{array}{cc}
c^{1 / 2} & 0 \\
0 & c^{-1 / 2}
\end{array}\right)\left(\begin{array}{cc}
\varphi_{1}^{0} & \varphi_{2}^{0} \\
\psi_{1}^{0} & \psi_{2}^{0}
\end{array}\right)
$$

If we choose $\varphi(P)=\psi\left(\bar{P}^{\tau}\right)$ then $c=1$. 
Now let us the rewrite formula for $J$ in an alternative form by choosing another basis in $S(\varphi, \psi)$ : let functions $\tilde{\varphi}$ and $\tilde{\psi}$ be defined by (3.4), where $Q_{\tilde{\varphi}}=\infty^{1}, Q_{\tilde{\psi}}=\infty^{2}$. Then it is easy to see that the function $\Psi(P)$ defined by (3.7) may be represented in the form

$$
\Psi(P)=\left(\begin{array}{cc}
-i \tilde{\varphi}(P)\left(\tilde{\varphi}_{2}^{\infty}\right)^{-1} & -i \tilde{\varphi}\left(P^{*}\right)\left(\tilde{\varphi}_{2}^{\infty}\right)^{-1} \\
i \tilde{\varphi}(P)\left(\tilde{\psi}_{1}^{\infty}\right)^{-1} & i \tilde{\psi}\left(P^{*}\right)\left(\tilde{\psi}_{1}^{\infty}\right)^{-1}
\end{array}\right)
$$

and

$$
J(y, \bar{y}, z, \bar{z})=i\left(\begin{array}{rr}
-\tilde{\varphi}_{1}^{0}\left(\tilde{\varphi}_{2}^{\infty}\right)^{-1} & -\tilde{\varphi}_{2}^{0}\left(\tilde{\varphi}_{2}^{\infty}\right)^{-1} \\
\tilde{\varphi}_{1}^{0}\left(\tilde{\psi}_{1}^{\infty}\right)^{-1} & \tilde{\varphi}_{2}^{0}\left(\tilde{\varphi}_{1}^{\infty}\right)^{-1}
\end{array}\right), \quad J_{\mathrm{ph}}=(\operatorname{det} J)^{-1 / 2} J .
$$

Formulae (3.4) and (3.6), (3.8), (3.10) or (3.11) give the finite-gap solution of selfduality equation.

Then we consider the solutions of the second- $\tau *$-class.

b. $S U(2) \tau *-$ Solutions. Now we define functions $\varphi$ and $\psi$ by slightly more complex formulae:

$$
\begin{aligned}
\varphi(P)= & \frac{\vartheta\left(\boldsymbol{U}(P)-\boldsymbol{U}(\boldsymbol{D})-\boldsymbol{U}\left(D_{0}\right)+\boldsymbol{U}\left(Q_{\varphi}\right)+\boldsymbol{B}+\boldsymbol{B}_{0}+\boldsymbol{b}-\boldsymbol{K}\right)}{\vartheta(\boldsymbol{U}(P)-\boldsymbol{U}(\boldsymbol{D})-\boldsymbol{K})} \\
& \times \exp \left\{W_{Q_{\varphi} D_{0}}(P)+W(P)+W_{0}(P)+w(P)\right\}, \\
\psi(P)= & \frac{\vartheta\left(\boldsymbol{U}(P)-\boldsymbol{U}(\boldsymbol{D})-\boldsymbol{U}\left(D_{0}\right)+\boldsymbol{U}\left(Q_{\psi}\right)+\boldsymbol{B}+\boldsymbol{B}_{0}+\boldsymbol{b}-\boldsymbol{K}\right)}{\vartheta(\boldsymbol{U}(P)-\boldsymbol{U}(\boldsymbol{D})-\boldsymbol{K})} \\
& \times \exp \left\{W_{Q_{\psi} D_{0}}(P)+W(P)+W_{0}(P)+w(P)\right\},
\end{aligned}
$$

where all objects except integral $W_{0}(P)$ with vector of $b$-periods $\boldsymbol{B}_{0}$ are the same as in (3.1) satisfying, however, to another reality condition: now $\left(D_{0}+\boldsymbol{D}\right)^{\tau *}=D_{0}+\boldsymbol{D}$; $\bar{w}\left(P^{\tau *}\right)=w(P)$; an integral of the third kind $W(P)$ has poles at points $\infty^{1}, \infty^{2}, O^{1}, O^{2}$ with the residue $\alpha,-\alpha,-\alpha, \alpha$ respectively. A normalized abelian integral of the third kind $W_{0}(P)$ is essentially a new object. It has poles at points $E_{i}, E_{i+g}$ with the residue $r_{i}= \pm 1 / 2$ and $F_{i}, F_{i+\mathrm{g}}$ with the residue $-r_{i}, i=0, \ldots, \mathfrak{g}-1$. For $W_{0}(P)$ to be completely defined, one also chooses the cuts between points $E_{i}$ and $F_{i}$, $i=0, \ldots, 2 \mathfrak{g}-1$ as shown in Fig. 1 ; such a choice provides the following behaviour of $W_{0}(P)$ under the anti-involution $\tau *$ :

$$
\bar{W}_{0}\left(\lambda^{\tau 2}\right)-W_{0}\left(\lambda^{1}\right)=\pi i / 2, \quad \bar{W}_{0}\left(\lambda^{21}\right)-W_{0}\left(\lambda^{2}\right)=-\pi i / 2,
$$

where $\lambda^{i}, i=1,2$ - point on the $i^{\text {th }}$ sheet of $\mathfrak{L}$ with projection $\lambda$ on $\mathbb{C}$.

It is not difficult to verify that the vector of $b$-periods of $W_{0}(P)$ has the following components:

$$
\begin{gathered}
\boldsymbol{B}_{0 \mathrm{~g}}=r_{0}= \pm 1 / 2 ; \quad \boldsymbol{B}_{0 k}=(1 / 2)\left(r_{k}+r_{0}\right) \\
\boldsymbol{B}_{0 k+\mathrm{g}}=(1 / 2)\left(-r_{k}+r_{0}\right), \quad k=1, \ldots, \mathfrak{g}-1 .
\end{gathered}
$$

Using the periodicity properties of the theta-function we see that the sign at $\pm 1 / 2$ is not essential and without loss of generality we can choose $\boldsymbol{B}_{0 \mathrm{~g}}=1 / 2 ; \boldsymbol{B}_{0 k}$, $\boldsymbol{B}_{0 k+\mathrm{g}}=0$ or $1 / 2, \boldsymbol{B}_{0 k}+\boldsymbol{B}_{0 k+\mathrm{g}}=1 / 2, k=1, \ldots, \mathfrak{g}-1$.

If we assume now $Q_{\varphi}=Q_{\psi}^{\tau *}$ then

$$
\varphi\left(\lambda^{1}\right)=c \bar{\psi}\left(\lambda^{2 \tau}\right) ; \quad \varphi\left(\lambda^{2}\right)=-c \bar{\psi}\left(\lambda^{1 \tau}\right) .
$$

Sign minus appears here due to the presence of the cuts $l_{i}$. 
Now matrix $\Phi(\lambda)$ has the following local behaviour at $\lambda=\mathrm{O}^{1}$ and $\lambda=\infty^{1}$ :

$$
\begin{gathered}
\Phi(\lambda)_{\lambda \sim O^{1}} \Phi_{0}\left(\begin{array}{cc}
\lambda^{\alpha} & 0 \\
0 & \lambda^{-\alpha}
\end{array}\right)(1+O(\lambda)), \\
\Phi(\lambda)_{\lambda \sim \infty^{1}}^{\sim} \Phi_{\infty}\left(\begin{array}{cc}
\lambda^{-\alpha} & 0 \\
0 & \lambda^{\alpha}
\end{array}\right)\left(1+O\left(\lambda^{-1}\right)\right),
\end{gathered}
$$

where as before

$$
\Phi_{0, \infty} \equiv\left(\begin{array}{cc}
\varphi_{1}^{0, \infty} & \varphi_{2}^{0, \infty} \\
\psi_{1}^{0, \infty} & \psi_{2}^{0, \infty}
\end{array}\right) .
$$

We choose matrix $C$ from (3.3) equal to $I$. Then in full analogy with (3.7) and

$$
\begin{gathered}
\Psi(\lambda)=\Phi_{\infty}^{-1} \Phi(\lambda), \\
J=\Phi_{\infty}^{-1} \Phi_{0}, \\
J_{\mathrm{ph}}=\left(\operatorname{det} \Phi_{\infty} / \operatorname{det} \Phi_{0}\right)^{1 / 2} \Phi_{\infty}^{-1} \Phi_{0} .
\end{gathered}
$$

Using (3.14) we can easily verify that $J_{\text {ph }}$ is hermitian and

$$
D=\left(\varphi_{1}^{0} \psi_{2}^{0}-\varphi_{2}^{0} \psi_{1}^{0}\right)^{-1 / 2}\left(\begin{array}{cc}
c^{-1 / 2} & 0 \\
0 & c^{1 / 2}
\end{array}\right)\left(\begin{array}{cc}
\varphi_{1}^{0} & \varphi_{2}^{0} \\
\psi_{1}^{0} & \psi_{2}^{0}
\end{array}\right)
$$

In analogy with $\tau$-solutions, another representation for $J$ may be obtained if we consider functions $\tilde{\varphi}$ and $\tilde{\psi}$ defined by (3.12) where $Q_{\tilde{\varphi}}=\infty^{2}, Q_{\tilde{\psi}}=\infty^{1} ; \tilde{\varphi}, \tilde{\psi}-$ another basis in $S(\varphi, \psi)$; in terms of $\tilde{\varphi}$ and $\tilde{\psi}$ formula for $J$ may be rewritten as

$$
J(y, \bar{y}, z, \bar{z})=\left(\begin{array}{cc}
\tilde{\varphi}_{1}^{0}\left(\tilde{\varphi}_{1}^{\infty}\right)^{-1} & \tilde{\varphi}_{2}^{0}\left(\tilde{\varphi}_{1}^{\infty}\right)^{-1} \\
\tilde{\varphi}_{1}^{0}\left(\tilde{\psi}_{2}^{\infty}\right)^{-1} & \tilde{\psi}_{2}^{0}\left(\tilde{\psi}_{2}^{\infty}\right)^{-1}
\end{array}\right), \quad J_{\mathrm{ph}}=(\operatorname{det} J)^{-1 / 2} J .
$$

Now we consider $\tau$ - and $\tau *$-solutions for the $S U(1,1)$ group.

c. $S U(1,1) \tau$ - and $\tau *$-Solutions. The Backlund transformation of Corrigan at al [24] establishes a bijective mapping between the manifolds of $S U(2)$ and $S U(1,1)$ self-dual fields: if

$$
J=\varphi^{-1}\left(\begin{array}{cc}
\varphi^{2}+\varrho \bar{\varrho} & -\bar{\varrho} \\
-\varrho & 1
\end{array}\right)(\varphi \in \mathbb{R}, \operatorname{det} J=1)
$$

is a $S U(2)$ solution of (2.3) then it is possible to construct a hermitian matrix

$$
\widetilde{J}=\left(\begin{array}{cc}
\varphi & -\varphi \omega \\
-\varphi \bar{\omega} & \varphi \omega \bar{\omega}-\varphi^{-1}
\end{array}\right), \operatorname{det} \tilde{J}=-1,
$$

where

$$
\begin{gathered}
-\varphi^{-2} \tilde{\nabla} \varrho=\bar{\nabla} \bar{\omega}, \\
\tilde{\nabla} \equiv\left(\partial_{z},-\partial_{y}\right), \quad \bar{\nabla} \equiv\left(\partial_{\bar{y}}, \partial_{\bar{z}}\right),
\end{gathered}
$$

which satisfies $(2.3)$ too and gives $S U(1,1)$ solution of self-duality equation.

This transformation was also derived by Tafel [10] by means of a traditional "dressing" procedure of ISM.

Hence it is natural to suppose that some formulae similar to the formulae for $S U(2)$ solutions from $p$. a and $\mathbf{b}$ may be written for the $S U(1,1)$ case also. Such formulae exist indeed. For example we will write down the explicit expressions for 
$S U(1,1) \tau *$-solutions. In full analogy with $S U(2) \tau *$-solutions

$$
J_{\mathrm{ph}}=\sigma_{3}\left(\operatorname{det} \Phi_{\infty} / \operatorname{det} \Phi_{0}\right)^{1 / 2} \Phi_{\infty}^{-1} \Phi_{0},
$$

where functions $\Phi_{\infty}$ and $\Phi_{0}$ are determined by (3.15) (functions $\varphi$ and $\psi$ are, of course, new). Formula for $J$ in analogy with (3.17) may be rewritten as

$$
J=\left(\begin{array}{rr}
\tilde{\varphi}_{1}^{0}\left(\tilde{\varphi}_{1}^{\infty}\right)^{-1} & \tilde{\varphi}_{2}^{0}\left(\tilde{\varphi}_{1}^{\infty}\right)^{-1} \\
-\tilde{\varphi}_{1}^{0}\left(\tilde{\psi}_{2}^{\infty}\right)^{-1} & -\tilde{\varphi}_{2}^{0}\left(\tilde{\psi}_{2}^{\infty}\right)^{-1}
\end{array}\right), J_{\mathrm{ph}}=(-\operatorname{det} J)^{-1 / 2} J,
$$

where functions $\tilde{\varphi}$ and $\tilde{\psi}$ are following:

$$
\begin{aligned}
& \tilde{\varphi}(P)=\frac{\vartheta\left(\boldsymbol{U}(P)-\boldsymbol{U}(\boldsymbol{D})-\boldsymbol{U}\left(D_{0}\right)+\boldsymbol{U}\left(\infty^{2}\right)+\boldsymbol{B}+\boldsymbol{b}-\boldsymbol{K}\right)}{\vartheta(\boldsymbol{U}(P)-\boldsymbol{U}(\boldsymbol{D})-\boldsymbol{K})} \exp \left\{W_{\infty^{2} D_{0}}(P)+W(P)+w(P)\right\}, \\
& \tilde{\psi}(P)=\frac{\vartheta\left(\boldsymbol{U}(P)-\boldsymbol{U}(\boldsymbol{D})-\boldsymbol{U}\left(D_{0}\right)+\boldsymbol{U}\left(\infty^{1}\right)+\boldsymbol{B}+\boldsymbol{b}-\boldsymbol{K}\right)}{\vartheta(\boldsymbol{U}(P)-\boldsymbol{U}(\boldsymbol{D})-\boldsymbol{K})} \exp \left\{W_{\infty^{1} D_{0}}(P)+W(P)+w(P)\right\},
\end{aligned}
$$

i.e. these expressions coincide with the expressions for $S U(2) \tau$-solutions but here the divisor $\boldsymbol{D}+D_{0}$ and integrals $W(P)$ and $w(P)$ are invariant upon the involution $\tau *$, i.e. coincide with those in (3.12) - formulae for $S U(2) \tau *$-solutions. The analytical properties of functions $\tilde{\varphi}$ and $\tilde{\psi}$ in this case are distinguished from those in the case of $S U(2) \tau *$-solutions only by omitting the integral $W_{0}(P)$, i.e. omitting the poles and the zeros of the degree $1 / 2$ at branch points.

The class of $S U(2) \tau *$-solutions may be obtained analogously; in this case the reverse is true - the poles and zeros of a degree $1 / 2$ at branch points must be introduced.

Taking into consideration similar analytical properties of $S U(1,1)$ and $S U(2)$ $\tau *$-solutions it is natural to suppose that they are connected by Backlund transformation (3.19). This hypothesis is true at least for one-instanton solution and the $5 N$-parametric 'tHooft family (see Sect. 4). The same seems to be true for the connection between classes of $S U(2)$ and $S U(1,1) \tau$-solutions.

Let us discuss some general properties of our solutions. They are similar to those of finite-gap solutions of Einstein's equations [16-18]. The dependence upon the dynamical variables in our solutions is given by the movement of our algebraic curve with branch points and the points of the divisor moving in a prescribed way in the modulispace of algebraic curves with marked points. Therefore any periodicity or almost periodicity is absent (in general case) in contrast to traditional finite-gap solutions of $\mathrm{KdV}$, NSh, SG and others. The class of our solutions is very broad: except arbitrary sufficiently smooth functions $h(u, v, t)$ which determine the solutions of "pole" system (2.5) by equations

$$
h(\lambda, \lambda y-\bar{z}, \lambda z+\bar{y})=0
$$

(functions $h$ may be different for different poles or branch points) we have functional parameters connected with integral $w(P)$ : it may have any number of poles with any constant residue.

The integral $w(P)$ corresponds to $U(1)$ (Abelian) background of our finite-gap solution: if $\mathfrak{g}=0$ then

$$
J_{\text {Abel }}=\left(\begin{array}{cc}
\exp \operatorname{Re}(w(\infty)-w(0)) & 0 \\
0 & \exp \operatorname{Re}(w(0)-w(\infty))
\end{array}\right)
$$


for such a form of $J(2.3)$ reduces to

$$
\Delta\left(\ln J_{\text {Abel }}\right)=0 \text {, }
$$

where $\Delta$-laplasian in $\mathbb{R}^{4}$.

Abelian background here is a full analog of a static background in the case of Einstein's equation. The linear structure on the manifold of solutions of (3.23) may be mapped on the manifold of finite-gap solutions with identical branch points and divisors of the poles - as a linear structure on the manifold of integrals $w(P)$ and their $b$-periods.

\section{Degeneration of Genus 1 Solutions}

Traditionally one way to obtain the multisoliton solutions of non-linear equations solvable by ISM was the degeneration of finite-gap solutions, then the corresponding Riemann surface is transferred into the surface of zero genus. For example, in $[16,18]$ by the degeneration of algebraic-geometric solutions of stationary axisymmetric Einstein's equations (which are reductions of solutions from Sect. 3 to the stationary axisymmetric case) the formulae describing the interaction of $N$ Kerr-NUT objects were obtained. This method of the construction of multisoliton solutions seems to be one of the most effective. Hence it is natural to suppose that by the degeneration of our finite-gap solutions we can obtain new representations for multi-instanton and multimonopole (i.e. "multisoliton") solutions. Here we will do one necessary step in this direction: the derivation of one-instanton solution both from $S U(2)$ and $S U(1,1) \tau *$-solutions. The one-monopole solution may be obtained in a similar way, but, as it was mentioned above, it requires some complication of general formulae - the introduction of essential singularities at $\infty^{1,2}$ and $O^{1,2}$; we omit it here for simplicity.

Let $g=1$. Consider a limit $E_{0}, F_{0} \rightarrow \lambda_{0}, E_{1}, F_{1} \rightarrow \lambda_{1}=\left(\lambda_{0}\right)^{-1}$ (Fig. 2). Points $\lambda_{0}$, $\lambda_{1}, D_{0}, D_{1}$ are solutions of the algebraic equation (3.22), where the function $h$ may be different for different points; the choice of these functions is very important. For one-instanton solution points $\lambda_{0}$ and $\lambda_{1}$ have to satisfy the equation

$$
\left(\lambda_{i} y-\bar{z}\right)\left(\lambda_{i} z-\bar{y}\right)-\kappa \lambda_{i}=0, \kappa \in \mathbb{R}, \kappa>0, i=0,1
$$

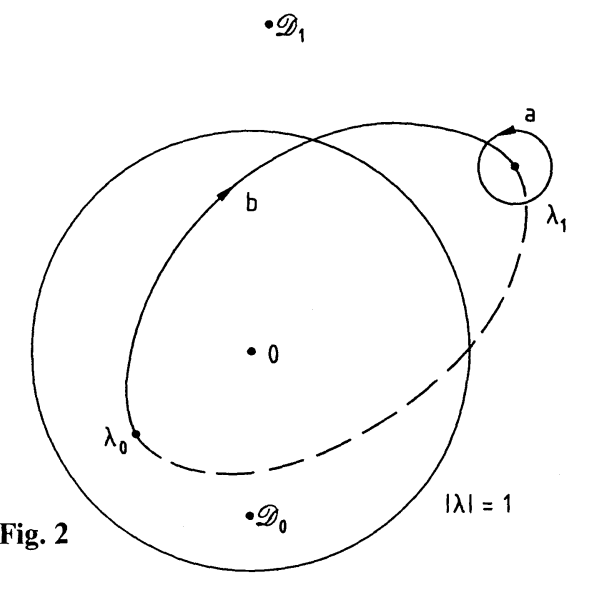


and points $D_{0}$ and $D_{1}$ - the equation

$$
\left(D_{i} y-\bar{z}\right)\left(D_{i} z+\bar{y}\right)=0, \quad i=0,1 .
$$

Note that such forms of pole trajectories were used in [9] to obtain the oneinstanton solution by the "dressing" method.

The behaviour of standard finite-gap objects in the limit $E_{i}, F_{i} \rightarrow \lambda_{i}, i=0,1$ is following $[16,17]$ :

$$
\begin{gathered}
d U(P)=(2 \pi i)^{-1} \frac{\lambda_{1}-\lambda_{0}}{\left(\lambda-\lambda_{0}\right)\left(\lambda-\lambda_{1}\right)} d \lambda \quad \text { if } P \text { lie on the first sheet; } \\
d U\left(P^{*}\right)=-d U(P) .
\end{gathered}
$$

For the matrix of $b$-periods of $\mathfrak{L}$ we have:

$$
\operatorname{Re}(i \mathbb{B})_{E_{i}, F_{i} \sim \lambda_{i}} \sim \frac{2}{\pi} \ln \left|F_{0}-F_{0}\right|+O(1) \text {, i.e. } \operatorname{Re}(i \mathbb{B}) \rightarrow-\infty .
$$

For vector $\boldsymbol{K}$ we have a well-known formula $K=(\mathbb{B}+1) / 2$. then

Consider the expression $U(P)-U(Q)$. If points $P$ and $Q$ lie on the first sheet of $\mathscr{Q}$

$$
U(P)-U(Q) \underset{E_{i}, F_{i} \rightarrow \lambda_{i}}{\longrightarrow} \frac{1}{2 \pi i} \ln \frac{\left(P-\lambda_{0}\right)\left(Q-\lambda_{1}\right)}{\left(P-\lambda_{1}\right)\left(Q-\lambda_{0}\right)} .
$$

If $P$ and $Q$ lie on the second sheet of $\mathfrak{E}$ then

$$
U(P)-U(Q) \underset{E_{i}, F_{i} \rightarrow \lambda_{i}}{\longrightarrow} \frac{1}{2 \pi i} \ln \frac{\left(P-\lambda_{1}\right)\left(Q-\lambda_{0}\right)}{\left(P-\lambda_{0}\right)\left(Q-\lambda_{1}\right)} .
$$

If $P$ and $Q$ lie on the different sheets of $\mathfrak{Q}$ then $U(P)-U(Q)$ depends upon the choice of the path between $P$ and $Q:$ if $P$ lies on the first sheet, $Q$ on the second sheet and path is passed through $\lambda_{0}$ then

$$
U(P)-U(Q)_{E_{i}, \widetilde{F_{i}} \rightarrow \lambda_{i}}-\frac{\mathbb{B}}{2}+O(1) .
$$

If the path is passed through $\lambda_{0}$ then

$$
U(P)-U(Q)_{E_{i}, \widetilde{F_{i}} \rightarrow \lambda_{i}} \frac{\mathbb{B}}{2}+O(1) .
$$

It remains to consider the behaviour of theta-function in our limit (see for details $[16,17])$ :

$$
\vartheta(z-\boldsymbol{K} \mid \mathbb{B})=\sum_{m \in \mathbb{Z}} \exp \left\{\pi i m^{2} \mathbb{B}+2 \pi i m\left(z-\frac{\mathbb{B}}{2}-\frac{1}{2}\right)\right\} \underset{\operatorname{Re}(i \mathbb{B}) \rightarrow-\infty}{\longrightarrow} 1-\exp (2 \pi i z),
$$

i.e. all our solutions in the degenerated case may be expressed in elementary functions of branch points and points of a divisor. Note that if $\operatorname{Im}(z)$ tends to $\pm \infty$ then $\vartheta(z-K \mid \mathbb{B})$ tends to 1 or $\infty$ respectively. Let's discuss also the behaviour of Abelian integrals of the third kind $W_{P Q}$ in our limit.

If $P$ and $Q$ lie on one sheet then on this sheet

$$
W_{P Q}(\lambda) \rightarrow \ln \frac{(\lambda-P)}{(\lambda-Q)}
$$


and on other sheet

$$
W_{P Q}(\lambda) \rightarrow 0 \text {. }
$$

Let $P$ and $Q$ lie on different sheets of $\mathfrak{L}(P$ - on the first sheet and $Q$ - on the second) and the cut between $P$ and $Q$ is passed through the branch cut $\left[E_{0}, F_{0}\right]$. Then in our limit we have on the first sheet

$$
W_{P Q}(\lambda) \rightarrow \ln \frac{(\lambda-P)}{\left(\lambda-\lambda_{0}\right)}
$$

and on the second sheet

$$
W_{P Q}(\lambda) \rightarrow \ln \frac{\left(\lambda-\lambda_{0}\right)}{(\lambda-Q)},
$$

i.e. one obtains additional poles at degenerated branch cuts.

For the beginning consider $S U(1,1) \tau *$-solution. Put $w(P)=0, \alpha=0$ and use (3.20), (3.21). Introducing new notations

one obtains

$$
\begin{gathered}
\hat{\varphi}(P) \stackrel{\text { def }}{\equiv} \tilde{\varphi}(P)\left(\tilde{\varphi}\left(\infty^{1}\right)\right)^{-1} \equiv \tilde{\varphi}(P)\left(\tilde{\varphi}_{1}^{\infty}\right)^{-1}, \\
\hat{\psi}(P) \stackrel{\text { def }}{\equiv} \tilde{\psi}(P)\left(\tilde{\psi}\left(\infty^{2}\right)\right)^{-1} \equiv \tilde{\psi}(P)\left(\tilde{\psi}_{2}^{\infty}\right)^{-1},
\end{gathered}
$$

$$
\begin{aligned}
\hat{\varphi}(P)= & \frac{\vartheta\left(\boldsymbol{U}(P)-\boldsymbol{U}\left(D_{0}\right)-\boldsymbol{U}\left(D_{1}\right)+\boldsymbol{U}\left(\infty^{2}\right)-\boldsymbol{K}\right) \vartheta\left(\boldsymbol{U}\left(\infty^{1}\right)-\boldsymbol{U}\left(D_{1}\right)-\boldsymbol{K}\right)}{\vartheta\left(\boldsymbol{U}(P)-\boldsymbol{U}\left(D_{1}\right)-\boldsymbol{K}\right) \vartheta\left(\boldsymbol{U}\left(\infty^{1}\right)-\boldsymbol{U}\left(D_{0}\right)-\boldsymbol{U}\left(D_{1}\right)+\boldsymbol{U}\left(\infty^{2}\right)-\boldsymbol{K}\right)} \\
& \times \exp \left\{\left.W_{\infty^{2} D_{0}}\right|_{\infty^{1}} ^{P}\right\}, \\
\hat{\psi}(P)= & \frac{\vartheta\left(\boldsymbol{U}(P)-\boldsymbol{U}\left(D_{0}\right)-\boldsymbol{U}\left(D_{1}\right)+\boldsymbol{U}\left(\infty^{1}\right)-\boldsymbol{K}\right) \vartheta\left(\boldsymbol{U}\left(\infty^{2}\right)-\boldsymbol{U}\left(D_{0}\right)-\boldsymbol{K}\right)}{\vartheta\left(\boldsymbol{U}(P)-\boldsymbol{U}\left(D_{0}\right)-\boldsymbol{K}\right) \vartheta\left(\boldsymbol{U}\left(\infty^{2}\right)-\boldsymbol{U}\left(D_{0}\right)-\boldsymbol{U}\left(D_{1}\right)+\boldsymbol{U}\left(\infty^{1}\right)-\boldsymbol{K}\right)} \\
& \times \exp \left\{\left.W_{\infty^{1} D_{1}}\right|_{\infty^{2}} ^{P}\right\} .
\end{aligned}
$$

Now using (4.5), (4.6), and (4.8) we can directly compute $\hat{\varphi}\left(O^{1}\right)$ and $\hat{\varphi}\left(O^{2}\right)$ because all arguments of degenerated theta-functions are finite:

$$
\begin{aligned}
\hat{\varphi}\left(O^{1}\right) & =\frac{\left(1-\frac{\lambda_{0}\left(\lambda_{1}-D_{1}\right)\left(\lambda_{0}-D_{0}\right)}{\lambda_{1}\left(\lambda_{0}-D_{1}\right)\left(\lambda_{1}-D_{0}\right)}\right)\left(1-\frac{\left(\lambda_{1}-D_{1}\right)}{\left(\lambda_{0}-D_{1}\right)}\right)}{\left(1-\frac{\left(\lambda_{1}-D_{1}\right)\left(\lambda_{0}-D_{0}\right)}{\left(\lambda_{0}-D_{1}\right)\left(\lambda_{1}-D_{0}\right)}\right)\left(1-\frac{\lambda_{0}\left(\lambda_{1}-D_{1}\right)}{\lambda_{1}\left(\lambda_{0}-D_{1}\right)}\right)} \\
& =\frac{\lambda_{0} \lambda_{1}+D_{0} D_{1}-D_{1}\left(\lambda_{0}+\lambda_{1}\right)}{D_{1}\left(D_{0}-D_{1}\right)}, \\
\hat{\psi}\left(O^{2}\right) & =\frac{\left(1-\frac{\lambda_{1}\left(\lambda_{0}-D_{0}\right)\left(\lambda_{1}-D_{1}\right)}{\lambda_{0}\left(\lambda_{1}-D_{0}\right)\left(\lambda_{0}-D_{1}\right)}\right)\left(1-\frac{\left(\lambda_{0}-D_{0}\right)}{\left(\lambda_{1}-D_{1}\right)}\right)}{\left(1-\frac{\left(\lambda_{1}-D_{1}\right)\left(\lambda_{0}-D_{0}\right)}{\left(\lambda_{0}-D_{1}\right)\left(\lambda_{1}-D_{0}\right)}\right)\left(1-\frac{\lambda_{1}\left(\lambda_{0}-D_{0}\right)}{\lambda_{0}\left(\lambda_{1}-D_{0}\right)}\right)} \\
& =\frac{\lambda_{0} \lambda_{1}+D_{0} D_{1}-D_{0}\left(\lambda_{0}+\lambda_{1}\right)}{D_{0}\left(D_{1}-D_{0}\right)} .
\end{aligned}
$$

To compute $\hat{\varphi}\left(O^{2}\right)$ and $\hat{\psi}\left(O^{1}\right)$ it is necessary to derive the behaviour of $\boldsymbol{U}\left(O^{2}\right)$ $-\boldsymbol{U}\left(D_{1}\right)$ and $\boldsymbol{U}\left(O^{1}\right)-\boldsymbol{U}\left(D_{0}\right)$. Due to the invariance of $\hat{\varphi}$ and $\hat{\psi}$ upon the change of 
the basic cycles we can choose the path between $O^{2}$ and $D_{1}$ passed through $\lambda_{1}$ and the path between $O^{1}$ and $D_{0}$ - through $\lambda_{2}$; then we see from (4.5) that corresponding theta-functions tend to unit.

Using (4.3), (4.5), and (4.7) one obtains:

$$
\begin{aligned}
\hat{\varphi}\left(O^{2}\right) & =\overline{\hat{\psi}\left(O^{1}\right)}=\frac{\left(D_{0}-\lambda_{0}\right)\left(1-\frac{\left(\lambda_{1}-D_{1}\right)}{\left(\lambda_{0}-D_{1}\right)}\right)}{D_{0}\left(1-\frac{\left(\lambda_{1}-D_{1}\right)\left(\lambda_{0}-D_{0}\right)}{\left(\lambda_{0}-D_{1}\right)\left(\lambda_{1}-D_{0}\right)}\right)} \\
& =\frac{\left(\lambda_{0}-D_{0}\right)\left(\lambda_{1}-D_{0}\right)}{D_{0}\left(D_{0}-D_{1}\right)} .
\end{aligned}
$$

Calculating $\operatorname{det} J=-\hat{\varphi}\left(O^{1}\right) \hat{\psi}\left(O^{2}\right)+\hat{\varphi}\left(O^{2}\right) \hat{\psi}\left(O^{1}\right)$ it is easy to obtain that it is equal to $-\left(\lambda_{0} \lambda_{1}\right) /\left(\mathrm{D}_{0} \mathrm{D}_{1}\right)$ and

$$
J_{\mathrm{ph}}=\left(\frac{D_{0} D_{1}}{\lambda_{0} \lambda_{1}}\right)^{1 / 2} J
$$

Now let's substitute in the expressions for $J_{\mathrm{ph}}$ functions $\lambda_{0,1}$ and $D_{0,1}$ satisfying the pole Eqs. (4.1) and (4.2) respectively. As a result one obtains

$$
J_{\mathrm{ph}}=\left(\begin{array}{cc}
\phi+1 & \phi \\
\phi & \phi-1
\end{array}\right),
$$

where $\phi(y, \bar{y}, z, \bar{z}) \equiv-\kappa /(y \bar{y}+z \bar{z})$.

After transformation $J_{\mathrm{ph}} \rightarrow \Omega J_{\mathrm{ph}} \Omega^{+}$, where $\Omega=\left(\begin{array}{rr}1 & -1 \\ 0 & 1\end{array}\right)$ we have

$$
J_{\mathrm{ph}}=\left(\begin{array}{cc}
0 & 1 \\
1 & -1+\phi
\end{array}\right) \text {. }
$$

This $S U(1,1)$ solution after Backlund transformation $[7,24]$ gives $S U(2)$ instanton with center in 0 and radius $\kappa^{1 / 2}$.

Then we consider the degeneration of genus $1 S U(2) \tau *$-solutions. Oneinstanton solution is obtained from (3.12), (3.17), where $\alpha=1 / 2$ and $w(P)=0$, i.e. in comparison with the one-instanton solution in $S U(1,1)$ framework here function $\Psi(\lambda)$ must include poles and zeros of the degree $1 / 2$ at branch points, $O^{1,2}$ and $\infty^{1,2}$.

We will not discuss here all details and write down only the final expressions for $J$ which appears in this case equal to $J_{\mathrm{ph}}$ :

$$
\begin{gathered}
\left(J_{\mathrm{ph}}\right)_{11}=\frac{\lambda_{0} \lambda_{1}\left(D_{1}-D_{0}\right)}{D_{1}\left(D_{0} D_{1}+\lambda_{0} \lambda_{1}-D_{0}\left(\lambda_{0}+\lambda_{1}\right)\right.}, \\
\left(J_{\mathrm{ph}}\right)_{12}=\overline{\left(J_{\mathrm{ph}}\right)_{21}}=-i \frac{\left(D_{0}-\lambda_{0}\right)\left(D_{0}-\lambda_{1}\right)}{D_{0}\left(D_{0} D_{1}+\lambda_{0} \lambda_{1}-D_{0}\left(\lambda_{0}+\lambda_{1}\right)\right.}, \\
\left(J_{\mathrm{ph}}\right)_{22}=\frac{\lambda_{0}+\lambda_{1}}{D_{0}}-\frac{\lambda_{0} \lambda_{1}\left(D_{1}-D_{0}\right)}{D_{0}\left(D_{0} D_{1}+\lambda_{0} \lambda_{1}-D_{0}\left(\lambda_{0}+\lambda_{1}\right)\right.} .
\end{gathered}
$$

Using the same pole and branch points trajectories as in $S U(1,1)$ case we find that $J_{\mathrm{ph}}$ may be represented in the form (3.18) where $\phi=1+\kappa / X$ and $\varrho=i(y \kappa) /(\bar{z} X)$; $X \equiv y \bar{y}+z \bar{z}$, i.e. $J$ corresponds to one-instanton solution. 
Note that formulae (4.9) set some solution of self-duality equation if $\lambda_{i}$ and $D_{i}$ are any solutions of the pole system; however, whether this subclass of solutions includes some solutions having a physical sense (except a one-instanton solution) or not is difficult to say.

Now we shall say a few words about the degeneration of multi-gap solutions with the genus more than one. It seems quite probable that in analogy with other integrable systems all multisoliton (i.e. $N$-instanton) solutions may be obtained by the degeneration of finite-gap solutions of genus $2 N-1$. The main problem here is to derive correct poles and branch points trajectories.

For example the $5 \mathrm{~N}$-parametric 'tHooft family is obtained from general formulae for $\tau *$-solutions; then $D_{i}$ are different roots of factorizable polynomial of degree $2 N$ :

$$
\prod_{i=0}^{N-1}\left(D_{k} y-\bar{z}-D_{k} y_{i}+\bar{z}_{i}\right)\left(D_{k} z+\bar{y}-D_{k} z_{i}-\bar{y}_{i}\right)=0, \quad k=0, \ldots, 2 N-1,
$$

and $\lambda_{i}$ are different roots of more complex expression:

$$
1-\sum_{i=0}^{N-1} \frac{\kappa_{i} \lambda_{k}}{\left(\lambda_{k} y-\bar{z}-\lambda_{k} y_{i}+\bar{z}_{i}\right)\left(\lambda_{k} z+\bar{y}-\lambda_{k} z_{i}-\bar{y}_{i}\right)}=0,
$$

where $\kappa_{i} \in \mathbb{R}, \kappa_{i}>0, y_{i}, z_{i} \in \mathbb{C}, i=0, \ldots, N-1-5 N$ real constants $y_{i}, z_{i}$ play the role of the center of $i^{\text {th }}$ instanton; $\kappa^{1 / 2}-$ of its radius.

To obtain in this way a general multi-instantion solution one must derive from a non-singularity requirement some more or less effective condition on two polynomials $h(\lambda, \lambda y-\bar{z}, \lambda z+\bar{y})$ upon three variables of degree $2 N$. (The fact that all degenerated branch cuts must be all roots of some polynomial of degree $2 N$ is trivial: in the opposite case the solution will not be one-sheet on $\mathbb{R}^{4}$; the same is true for $2 \mathrm{~N}$ points of a divisor.)

It seems that $\tau$-solutions in the degenerated case give only quite trivial limits; therefore it would be desirable to investigate them in the non-degenerated situation.

\section{Conclusions}

In this paper, following [20], we apply to $S U(2)$ and $S U(1,1)$ self-duality equations the method of finite-gap integration developed for the majority of the equations solvable by inverse scattering method. This seems to be the first example of a fourdimensional equation admitting the finite-gap construction of the solutions. Finite-gap solutions of the stationary axisymmetric vacuum Einstein's equation are reductions of the present solutions to the stationary axisymmetric case. Therefore most general properties of our solutions are the same as in the case of Einstein's equation: the dynamics is set by the deformation of the Riemann surface with the prescribed dependence of branch points and points of a non-special divisor in the moduli space of algebraic curves; our solutions have no periodicity properties. The asymptotic properties of finite-gap solutions in this case seem similar to those of the degenerated-multisoliton solutions.

In addition to classes of $\tau *$-solutions for $S U(2)$ and $S U(1,1)$ groups [20] one obtains here classes of $\tau$-solutions for these groups. The corresponding classes of $S U(2)$ and $S U(1,1)$ solutions seem connected by a simple "dressing" procedure [10] initially derived by Corrigan et al. [24]. This connection is verified in Sect. 4 for 
one-instanton solutions. We also described how to obtain in our framework the $5 N$-parametric 'tHooft family of instantons. It seems that our solutions in the degenerated case include all $8 N-3$-parametric family of instantons, but to describe this family it is necessary to derive some non-singularity condition for our solutions in terms of the trajectories of the poles and branch points.

After a simple generalization the class of our $\tau *$-solutions includes all multimonopole configurations. The derivation of non-singularity conditions in this case seems to be simpler than for instantons because all trajectories of the poles and branch points are set by the polynomials upon two variables (instead of the polynomials upon three variables in the case of instantons).

Note that not only monopole- and instanton-type solutions may be interesting from the physical point of view. For example, it's possible that there is some physical sense of "torons" - solutions with half topological charge [25], string-type solutions and so on. Therefore it would be interesting to analyse the properties of the simplest genus 1 non-degenerated solutions at least. We intend to carry out such analysis in further publications.

Acknowledgements. I am grateful to Prof. V. B. Matveev for his encouragement and attention to this work and Prof. A. R. Its for helpful stimulating discussions.

\section{References}

1. Manin, Yu.I.: Gauge fields and complex geometry. Moscow: Science 1984

2. Marathe, K.B., Martucci, G.: J. Geom. and Phys. 6, 1-106 (1989)

3. Rajaraman, R.: Solitons and instantons. Amsterdam: North-Holland 1982

4. Belavin, A.A., Zakharov, V.E.: Pisma JETP 25, 603-607 (1977)

5. Atiyah, M.F., Hitchin, N.J., Drinfeld, V.B., Manin, Yu.I.: Phys. Lett. 65A, 185 (1978)

6. Forgacs, P.: In: Non-linear equations in classical and quantum field theory. Sanches, N. (ed.). Berlin, Heidelberg, New York: Springer 1985

7. Forgacs, P., Horvath, Z., Palla, L.: Phys. Rev. D 23, 1876-1879 (1981)

8. Chau, L.-L., Prasad, M.K., Sinha, A.: Phys. Rev. D 24, 1574-1580 (1981)

9. Chau, L.-L., Shau, J.S., Yen, H.C.: A generalized Backlund transformation for the (supersymmetric) self-dual Yang-Mills fields. (Preprint) UCD-88-28

10. Tafel, J.: J. Math. Phys. (to appear)

11. Dubrovin, B.A., Matveev, V.B., Novikov, S.P.: UMN. 31, 55-137 (1976)

12. Matveev, V.B.: Abelian functions and solitons. (Preprint) University of Wroclaw 373 (1976)

13. Dubrovin, B.A.: UMN. 36, 11-80 (1981)

14. Its, A.R.: Zapiski nauch. sem. LOMI 133, 113-125 (1984)

15. Bikbayev, R.F., Bobenko, A.I., Its, A.R.: DAN USSR 272, 1293-1298 (1983)

16. Korotkin, D.A.: Theor. Math. Phys. 77, 25-41 (1988)

17. Korotkin, D.A., Matveev, V.B.: In: Some topics on inverse problems. Sabatier, P. (ed.). Singapore: World Scientific 1988

18. Korotkin, D.A., Matveev, V.B.: Algebra Analysis 1, 77-102 (1989) (English translation to be published in vol. 1 of Leningrad Mathematical Journal)

19. Burtzev, S.P., Zakharov, V.E., Mikhailov, A.V.: Theor. Math. Phys. 70, 323-335 (1987)

20. Korotkin, D.A.: Math. sbornik 181, N7, 923-933 (1990)

21. Krichever, I.M.: Funct. Anal. Appl. 13, 81-82 (1979)

22. Cherednik, I.: DAN USSR 246, 575-578 (1979)

23. Ercolani, N., Sinha, A.: Commun. Math. Phys. 125, 385-416 (1989)

24. Corrigan, E., Fairlie, D.B., Goddard, P., Yates, R.G.: Commun. Math. Phys. 58, 223-240 (1978)

25. Zhitnitsky, A.R.: JETP 95, 24-34 (1989); 96, 1167-1180 (1989) 